\title{
Histological findings in infants with Gastrointestinal food allergy are associated with specific gastrointestinal symptoms; retrospective review from a tertiary centre
}

\author{
Neil Shah ${ }^{1,3^{*}}{ }$, Ru-Xin Melanie Foong ${ }^{1 \dagger}$, Osvaldo Borrelli ${ }^{1}$, Eleni Volonaki ${ }^{1}$, Robert Dziubak ${ }^{1}$, Rosan Meyer ${ }^{1}$,
} Mamoun Elawad ${ }^{1}$ and Neil J. Sebire ${ }^{2}$

\begin{abstract}
Background: Gastrointestinal food allergy (GIFA) occurs in 2 to $4 \%$ of children, the majority of whom are infants ( $<1$ year of age). Although endoscopy is considered the gold standard for diagnosing GIFA, it is invasive and requires general anaesthesia. Therefore, we aimed to investigate whether in infants with GIFA, gastrointestinal symptoms predict histological findings in order to help optimise the care pathway for such patients.

Methods: All infants $<1$ year of age over a 20 year period who underwent an endoscopic procedure gastroscopy or colonoscopy for GIFA were evaluated for the study. Symptoms at presentation were reviewed and compared with mucosal biopsy histological findings, which were initially broadly classified for study purposes as "Normal" or "Abnormal" (defined as the presence of any mucosal inflammation by the reporting pathologist at the time of biopsy).

Results: Of a total of 1319 cases, 544 fitted the inclusion criteria. $62 \%$ of mucosal biopsy series in this group were reported as abnormal. Infants presenting with diarrhoea, rectal (PR) bleeding, irritability and urticaria in any combination had a probability $>85 \%(O R>5.67)$ of having abnormal histological findings compared to those without. Those with isolated PR bleeding or diarrhoea were associated with $74 \%$ and $68 \%$ probability (OR: 2.85 and 2.13) of an abnormal biopsy, respectively. Conversely, children presenting with faltering growth or reflux/vomiting showed any abnormal mucosal histology in only $50.8 \%$ and $45.3 \%$ (OR: 1.04 and 0.82 ) respectively.

Conclusions: Food allergy may occur in very young children and is difficult to diagnose. Since endoscopy in infants has significant risks, stratification of decision-making may be aided by symptoms. At least one mucosal biopsy demonstrated an abnormal finding in around half of cases in this selected population. Infants presenting with diarrhoea, PR bleeding, urticaria and irritability are most likely to demonstrate abnormal histological findings.
\end{abstract}

Keywords: Endoscopy, Infant, Food allergy, Biopsy, Histopathology, Eosinophil

\section{Background}

Gastrointestinal food allergy (GIFA) is increasing in prevalence and usually affects very young children [1]. Approximately $2-4 \%$ of children between the ages of 0 3 years are diagnosed with food allergy $[2,3]$ and up to $60 \%$ of these children display gastrointestinal symptoms

\footnotetext{
* Correspondence: neil.shah@gosh.nhs.uk

${ }^{\dagger}$ Equal contributors

'Paediatric Gastroenterology Department, Great Ormond Street Hospital, London WC1N 3JH, United Kingdom

${ }^{3}$ Institute of Child Health/UCL, London WC1N 1EH, UK

Full list of author information is available at the end of the article
}

such as abdominal pain, poor appetite, vomiting and diarrhoea. Other children may present with symptoms affecting skin, such as eczema, catarrhal problems or even anaphylaxis. Clinically, symptoms are often very pronounced and warrant investigations to eliminate other diagnoses before food allergy is considered $[2,4,5]$. Normally, the mucosal barrier in the gastrointestinal (GI) tract develops an "oral tolerance" to food antigens ingested [6]. However, in children with food allergy, this mechanism is believed to fail, resulting in allergic sensitisation and elicitation of allergy-type responses [6, 7]. This reaction, which

\section{() Biomed Central}


can manifest as a wide range of different symptoms, can be classified as immunoglobulin E (IgE)-mediated allergy, non-IgE mediated allergy or mixed IgE and non-IgE allergy [8]. Gastrointestinal food allergies (GIFA) are generally considered as non-IgE mediated, but eosinophilic dominant gastrointestinal disorders may be mixed $\operatorname{IgE}$ and non-IgE allergies. The most common age of presentation of non-IgE mediated allergies affecting the gut is in children under the age of one year, with cow's milk, soy protein, hens' egg and wheat being the most frequent causative foods $[2,4,5,9,10]$.

The immunopathology of non-IgE mediated GIFA is still not fully understood, which makes diagnosis and management difficult, often requiring an elimination diet followed by food challenge $[8,11]$. Endoscopy and biopsy has become increasingly important, with some considering endoscopic biopsy as the gold standard since it is relatively objective and may provide information regarding possible mechanisms. [4, 7] For example, in eosinophilic oesophagitis, the histological appearance defines the diagnosis [12]. However, endoscopy for very young children is often limited to specialised centres and involves general anaesthesia, requiring administration by paediatric anaesthetists, and procedural risks such as intestinal perforation $[13,14]$. There are no studies investigating gastrointestinal symptoms in relation to histological features in infant GIFA [15]. Hence, the aim of this study was to investigate whether specific symptoms are associated with abnormal histological findings in endoscopic biopsies obtained from children with GIFA in order to optimise care pathway decision making.

\section{Methods}

Routinely collected data was reviewed from children under the age of one-year referred to a tertiary paediatric gastroenterology centre during the study period (June 1987 to August 2007), who had undergone endoscopic biopsy. Jejunal biopsies performed by the now historical procedure of Crosby capsule (common in the early years of our study) were excluded, and we also excluded children biopsied for other indications unrelated to GIFA. For all cases clinical symptoms were assessed in relation to histopathological findings based on contemporaneous biopsy reports. A single researcher extracted data according to predefined objective criteria.

All biopsies were reported by specialist paediatric histopathologists from the same tertiary centre. For the purposes of this study, histopathological findings were coded as either "Normal" or "Abnormal" (presence of any significant abnormal finding at any biopsy site including acute or chronic inflammation, with or without increased mucosal eosinophil density [16], or other pathologies such as partial villous atrophy or Helicobacter pylori see Fig. 1). Chronic inflammation with predominantly excess mucosal eosinophil density was considered most suggestive of food allergy in this cohort of young children [12, 17, 18].

Data were analysed using IBM SPSS Statistics for Windows, Version 22 (Armonk, NY). Continuous variables

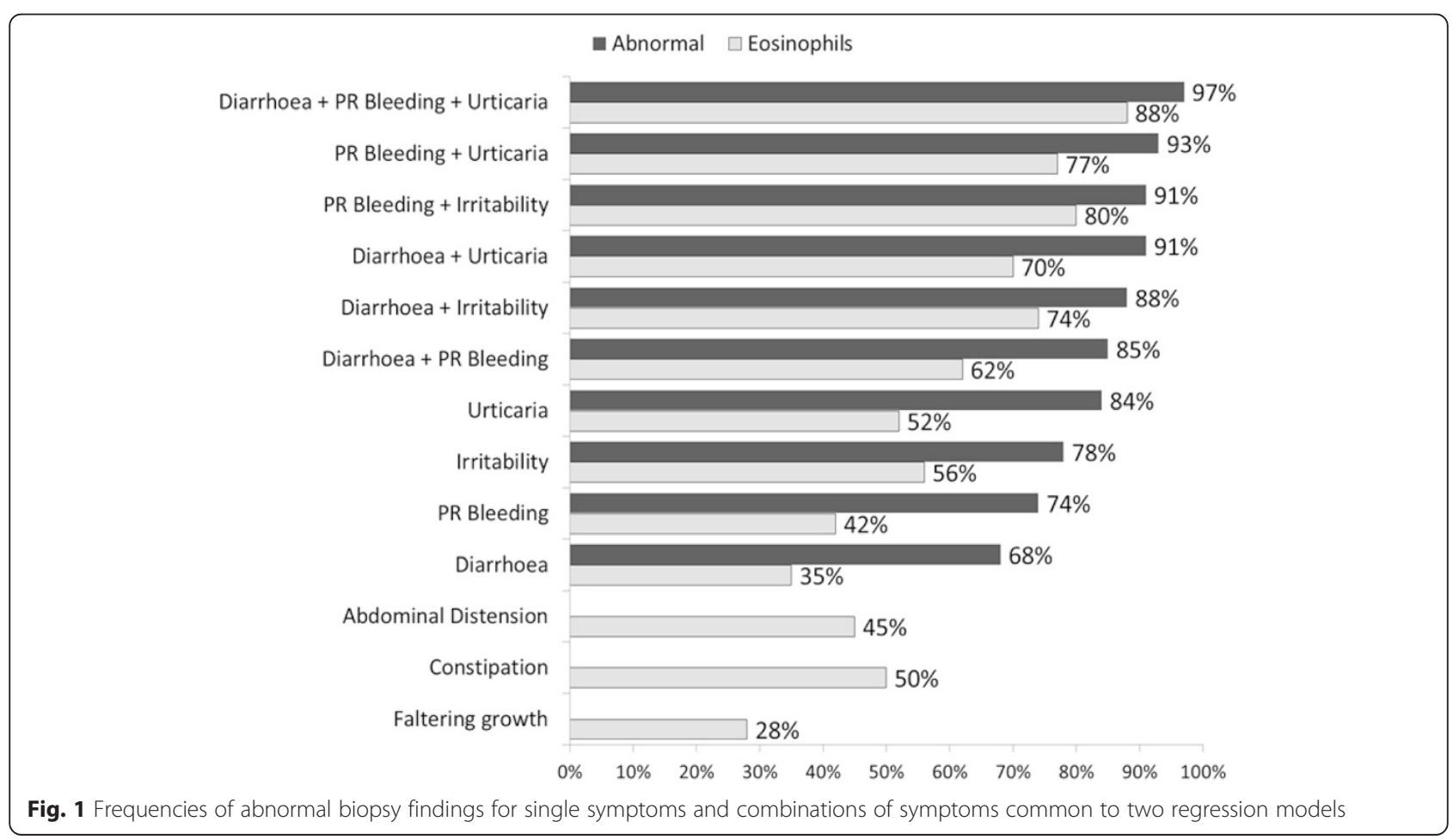


were presented as medians with interquartile ranges and categorical variables as frequencies and percentages. Mann-Whitney $U$ test and chi-square test were used to examine the differences between groups.

For all symptoms in isolation (in cases when the patient presented with only one symptom) Positive Predictive Values (PPV) of abnormal biopsy findings were calculated. Multiple logistic regression was used to investigate the relationship between biopsy findings and symptoms with adjustment for potential confounders of age and gender. Based on logistic regression models, probabilities of abnormal biopsy findings were calculated for combinations of symptoms using the median age. Goodness of fit of logistic regression models was based on Hosmer-Lemeshow test. All tests were two-tailed and significance level was set to 0.05 . The study was approved by the local Research Ethics Committee (Bloomsbury REC). All data was retrospective and identified by study number only and individual patient consent was not required for data inclusion. The study conformed to the Helsinki declaration regarding research performance.

\section{Results}

Of 1319 infants undergoing endoscopic biopsies, 318 were excluded due to insufficient clinical information, 265 due to being Crosby capsule biopsies and 60 due to specific non GIFA indications (congenital diarrhoea, autoimmune enteropathy, graft-versus-host disease, tufting enteropathy and disaccharidase deficiency), leaving 676 patients who met the inclusion criteria. Some patients had multiple endoscopies and repeat biopsies, 122 in total, which were also excluded and only the initial presentation biopsy included. 554 endoscopic biopsy series were therefore included. Fifty-one per cent (285/ 554 ) were male, median age 7 months (IQR $=0.2-12$ months). Overall, $62 \%(344 / 554)$ had abnormal mucosal biopsy findings. The median age of those with abnormal biopsy was significantly lower than those with normal findings (median 6.6 months versus median 7.5 months, $\mathrm{P}<0.001)$.

The most common presenting symptoms as indications for endoscopy were reflux/vomiting (40\%), faltering growth (37\%), diarrhoea (35\%) and rectal (PR) bleeding (12\%). 309 (56\%) patients presented with one symptom, 190 (34\%) with two, 49 (9\%) with three and six patients $(1 \%)$ with four symptoms. Positive predictive values (PPV) for symptoms based on the patients who presented with one symptom $(n=309)$ are shown in Table 1.

Diarrhoea was associated with a significantly greater frequency of abnormal histological findings than faltering growth (70.8 \% vs. $50.8 \%, p=0.018)$ or reflux (70.8 \% vs. $45.3 \%, p=0.001)$. PR bleeding was associated with a significantly greater rate of abnormal histological findings than faltering growth $(74.2 \%$ vs. $50.8 \%, p=$ $0.031)$ or reflux $(74.2 \%$ vs. $45.3 \%, p=0.004)$. There were no significant differences between the frequency of abnormal biopsies between those presenting with diarrhoea and PR Bleeding, $(p=0.728)$ or those presenting with reflux and faltering growth, $(p=0.484)$.

Multiple logistic regression models were used to assess the frequency of any abnormal biopsy findings with combinations of symptoms (Hosmer-Lemeshow $p=$ 0.373 ), as well as the probability of increased mucosal eosinophil density (Hosmer-Lemeshow $p=0.413$ ), adjusted for differences in age and gender. Diarrhoea $(p<$ $0.001)$, PR bleeding $(p<0.01)$, irritability $(p<0.05)$ and urticaria $(p<0.05)$ were significantly associated with both abnormal biopsy and excess eosinophils (Table 2). The site, Oesophagus, stomach, duodenum or colon, of the abnormal findings are shown in Table 3. Faltering growth, constipation and abdominal distension were also predictors of finding eosinophils in a biopsy $(p<$ $0.05, p<0.01, p<0.05$ respectively; Table 2). Age was an important confounding factor as we found a higher probability of an abnormal biopsy in younger children. Among others, reflux/vomiting was a poor predictor; therefore it was excluded from the models. Similarly, gender was not a significant confounding factor.

Specific symptom combinations were more likely to have biopsies with excess eosinophils present. For example, children who presented with a combination of diarrhoea, PR bleeding and urticaria had an $88 \%$ frequency of excess eosinophils.

\section{Discussion}

This is the first large study to examine whether specific symptoms at presentation in very young children are associated with abnormal endoscopic biopsy findings in children being assessed for GIFA. The findings demonstrate that younger infants are more likely to have abnormal mucosal histological findings, and those presenting with specific combinations of symptoms are associated with high frequency of abnormal mucosal biopsy findings, including increased mucosal eosinophil density. In the current patient population, first presentation of GI symptoms occurred at around five months of age. However, it is likely that this represents a highly selected group referred to a specialist centre who are likely to have been experiencing more severe symptoms and hence were evaluated earlier in life than the general population and were all deemed to have symptoms of sufficient severity to warrant endoscopic examination. The risks of undergoing a general anaesthetic procedure and associated potential complications involved in performing endoscopy in very young infants as well as the impact on families are important considerations when deciding on whether to perform an endoscopy $[13,14,19]$. 
Table 1 Positive Predictive Values (PPV) of abnormal mucosal biopsies in infants being assessed for GIFA based on a single presenting gastrointestinal symptom based on 309/554 patients who presented with one symptom only

\begin{tabular}{|c|c|c|c|c|c|c|}
\hline & Number & Percent & $\begin{array}{l}\text { Isolated increased mucosal eosinophil density } \\
n\end{array}$ & PPV & $\begin{array}{l}\text { Any abnormal biopsy } \\
n\end{array}$ & PPV \\
\hline Reflux/vomiting & 117 & $37.9 \%$ & 19 & $16.2 \%$ & 53 & $45.3 \%$ \\
\hline Diarrhoea & 72 & $23.3 \%$ & 30 & $41.7 \%$ & 51 & $70.8 \%$ \\
\hline Faltering growth & 61 & $19.7 \%$ & 19 & $31.1 \%$ & 31 & $50.8 \%$ \\
\hline PR Bleeding & 31 & $10.0 \%$ & 16 & $51.6 \%$ & 23 & $74.2 \%$ \\
\hline Haematemesis & 7 & $2.3 \%$ & 1 & $14.3 \%$ & 4 & $57.1 \%$ \\
\hline Constipation & 6 & $1.9 \%$ & 3 & $50.0 \%$ & 3 & $50.0 \%$ \\
\hline Feeding difficulties & 4 & $1.3 \%$ & 0 & $0.0 \%$ & 0 & $0.0 \%$ \\
\hline Irritability & 4 & $1.3 \%$ & 1 & $25.0 \%$ & 2 & $50.0 \%$ \\
\hline Anaemia & 2 & $0.6 \%$ & 0 & $0.0 \%$ & 0 & $0.0 \%$ \\
\hline Hypoalbuminaemia & 2 & $0.6 \%$ & 0 & $0.0 \%$ & 2 & $100.0 \%$ \\
\hline Abdominal distension & 2 & $0.6 \%$ & 1 & $50.0 \%$ & 1 & $50.0 \%$ \\
\hline Recurrent Abdominal pain & 1 & $0.3 \%$ & 0 & $0.0 \%$ & 1 & $100.0 \%$ \\
\hline
\end{tabular}

The most common symptoms were diarrhoea, reflux/ vomiting, PR bleeding and faltering growth. Of these, if isolated, infants presenting with diarrhoea or PR bleeding, had an approximate $70 \%$ probability of a histologically abnormal biopsy. However, infants who presented with combinations of diarrhoea, PR bleeding, irritability and urticaria were both more likely to have abnormal biopsy findings and also more likely to demonstrate increased mucosal eosinophil density. For example, almost $90 \%$ of those presenting with diarrhoea and irritability had an abnormal biopsy. There were few patients who presented with irritability in isolation (1.3\%). However, it is possible that parents and clinicians underreport this symptom especially in the presence of other more common and recognisable symptoms. [20, 21] The frequency of an abnormal biopsy was lower for infants presenting with reflux/vomiting (45\%) and faltering growth (51 \%).

Food allergic diagnoses are classified according to the site and severity of inflammation, which influences the presenting symptoms. With gastrointestinal mucosal disease that is identified by endoscopic biopsy there is a close spatial relationship of inflammatory mediators known to be released by mucosal inflammatory cells and enteric nerves [2]. The exact mechanisms of the manifestations of gastrointestinal symptoms are slowly being unravelled with the concept of paracrine immune interaction on the enteric nervous system, being known as a neuro-immune interaction [22, 23] leading to the disturbed motility and symptoms seen in GIFA such as reflux, diarrhoea or constipation. Much workstill needs to be done to fully explain how these symptoms develop and respond to dietary or anti-inflammatory measures.

Limitations of the study are related to the retrospective nature of data collected over a long time period with possible associated variation in the clinical suspicion of GIFA and management of such infants by endoscopic examination and biopsy. In GIFA, despite adherence to the diagnosis only being made by clinicians in out unit

Table 2 Multiple logistic regression models for association with abnormal mucosal biopsies in infants being assessed for GIFA

\begin{tabular}{|c|c|c|c|c|}
\hline \multirow[b]{2}{*}{ Variables in the model } & \multicolumn{2}{|c|}{ Regression model for excess eosinophils in biopsy } & \multicolumn{2}{|c|}{ Regression model for any Abnormal Biopsy } \\
\hline & B & $p$-value & B & $p$-value \\
\hline Constant & -0.96 & 0.001 & 0.49 & 0.05 \\
\hline Age (months) & -0.07 & 0.033 & -0.06 & 0.041 \\
\hline Urticaria & 1.50 & 0.007 & 1.59 & 0.038 \\
\hline Irritability & 1.69 & $<0.001$ & 1.22 & 0.018 \\
\hline PR Bleeding & 1.12 & $<0.001$ & 1.00 & 0.002 \\
\hline Diarrhoea & 0.79 & $<0.001$ & 0.71 & $<0.001$ \\
\hline Constipation & 1.42 & 0.002 & $\mathrm{n} / \mathrm{a}$ & $\mathrm{n} / \mathrm{a}$ \\
\hline Abdominal Distension & 1.24 & 0.045 & $n / a$ & $\mathrm{n} / \mathrm{a}$ \\
\hline Faltering growth & 0.49 & 0.016 & $\mathrm{n} / \mathrm{a}$ & n/a \\
\hline
\end{tabular}


Table 3 Symptoms related to the site of abnormal findings in cases with only a single site affected

\begin{tabular}{lllllllll}
\hline & Oesophagus $(n=61)$ & Percent & Stomach $(n=16)$ & Percent & Duodenum $(n=50)$ & Percent & Colon $(n=44)$ & Percent \\
\hline RefluxNomiting & 44 & $72 \%$ & 5 & $31 \%$ & 19 & $38 \%$ & 15 & $34 \%$ \\
FTT & 18 & $30 \%$ & 5 & $31 \%$ & 26 & $52 \%$ & 18 & $41 \%$ \\
Diarrhoea & 5 & $8 \%$ & 6 & $38 \%$ & 19 & $38 \%$ & 18 & $41 \%$ \\
PR Bleeding & 3 & $5 \%$ & 0 & $0 \%$ & 5 & $10 \%$ & 10 & $23 \%$ \\
Constipation & 0 & $0 \%$ & 2 & $13 \%$ & 3 & $6 \%$ & 8 & 2 \\
Anaemia & 0 & $0 \%$ & 0 & $0 \%$ & 0 & $0 \%$ & 2 \\
Feed Diff & 2 & $3 \%$ & 2 & $13 \%$ & 3 & $6 \%$ & 2 \\
Mouth Ulcers & 0 & $0 \%$ & 0 & $0 \%$ & 0 & $0 \%$ & 0 \\
Rash & 1 & $2 \%$ & 0 & $0 \%$ & 3 & $6 \%$ & 2 \\
Iritability & 2 & $3 \%$ & 1 & $6 \%$ & 4 & $8 \%$ & 5 \\
Haematemesis & 6 & $10 \%$ & 3 & $19 \%$ & 1 & $2 \%$ & 0 \\
Hypoalbuminaemia & 0 & $0 \%$ & 1 & $6 \%$ & 2 & $4 \%$ & 1 \\
Abd. Distension & 1 & $2 \%$ & 0 & $0 \%$ & 1 & $2 \%$ & 5 \\
RAP & 1 & $2 \%$ & 0 & $0 \%$ & 0 & $0 \%$ & 3 \\
\hline
\end{tabular}

following elimination diet of major dietary antigens (Diary, egg, soya and wheat usually) with clinical improvement and subsequent reappearance of symptoms on rechallenge, the diagnosis remains subjective. The symptoms can be delayed and is unblended and subject to parental reporting. Furthermore, even with such a large dataset, for the purposes of this study we have classified mucosal biopsy findings broadly into normal versus abnormal, (with the only subcategory being those with apparently isolated increased mucosal eosinophil density at any site since this has been suggested as the most characteristic feature of GIFA) [12, 24]. More detailed sub-analysis of the relationship between other specific histological findings and their combinations with symptoms is not possible in this dataset and much larger numbers of cases, all of whom undergo multiple biopsies from small and large intestinal sites, would be required, but is unlikely to be available.

The clinical decision regarding whether an infant requires endoscopic examination and biopsy for diagnosis of food allergy can be difficult, since the procedure in this age group requires general anaesthesia with associated risks. The current data demonstrates that specific symptom patterns at presentation are associated with varying yield of abnormal mucosal histological findings, in particular, infants who experience diarrhoea, PR bleeding, irritability and urticaria having a high frequency of abnormal biopsies. This information may aid the decision making process for young children presenting with probable food allergy.

\section{Conclusions}

Gastrointestinal food allergy (GIFA), may present with a wide variety of symptoms in the first year of life and specific symptom patterns at presentation are associated with varying yield of abnormal mucosal histological findings at endoscopic biopsy. Infants who experience diarrhoea, PR bleeding, irritability and urticaria have a high frequency of abnormal gastrointestinal mucosal biopsies, including prominent mucosal eosinophils.

\section{Competing interests}

Dr Shah has performed consultancy work for Mead Johnson Nutrition, unrelated to this project. The other authors declare that they have no competing interests and have not received reimbursements, fees, funding, or salary from an organization that may in any way gain or lose financially from the publication of this manuscript, either now or in the future.

\section{Authors' contributions}

NS, ME and NJS conceived and planned the study. RMF, EV, RD and RM performed the data extraction and analysis. All authors participated in drafting of the manuscript and read and approved the final manuscript.

\section{Acknowledgements}

NJS is part supported by an NIHR Senior Investigator award and the NIHR GOSH BRC. This report is independent research and the views expressed in this publication are those of the authors and not necessarily those of the $\mathrm{NHS}$, the NIHR or the Department of Health.

\section{Author details}

${ }^{1}$ Paediatric Gastroenterology Department, Great Ormond Street Hospital, London WC1N 3JH, United Kingdom. '2Histopathology Department, Great Ormond Street Hospital, London, United Kingdom. ${ }^{3}$ Institute of Child Health/ UCL, London WC1N 1EH, UK.

Received: 10 February 2015 Accepted: 5 June 2015

Published online: 16 June 2015

\section{References}

1. Prescott SL, Pawankar R, Allen KJ, et al. A global survey of changing patterns of food allergy burden in children. World Allergy Organ J. 2013;6:21.

2. Meyer R, Schwarz C, Shah N. A Review of the Diagnosis and Management of Food-induced Gastrointestinal Allergies. Curr Allergy Clin Immunol. 2012;25:10-7.

3. Venter C, Pereira B, Voigt $K$, et al. Prevalence and cumulative incidence of food hypersensitivity in the first 3 years of life. Allergy. 2008;63:354-9. 
4. Maloney J, Nowak-Wegrzyn A. Educational clinical case series for pediatric allergy and immunology: Allergic proctocolitis, food protein-induced enterocolitis syndrome and allergic eosinophilic gastroenteritis with protein-losing gastroenteropathy as manifestations of non-lgE-mediated cow's milk allergy. Pediatr Allerg Immunol. 2007;18:360-7.

5. Meyer R, Fleming C, Dominguez-Ortega G, et al. Manifestations of food protein induced gastrointestinal allergies presenting to a single tertiary paediatric gastroenterology unit. World Allergy Organ J. 2013;6:13-6.

6. Dupont C. Food allergy: recent Advances in Pathophysiology and Diagnosis. Ann Nutr Metab. 2011;59:8-18.

7. Vickery BP, Chin S, Burks AW. Pathophysiology of Food Allergy. Pediatr Clin North Am. 2011;58:363-76.

8. Boyce JA, Assaad A, Burks AW, et al. Guidelines for the Diagnosis and Management of Food Allergy in the United States: Summary of the NIAIDSponsored Expert Panel Report. Nutr Res. 2011;31:61-75.

9. Husby S. Food Allergy as Seen by a Paediatric Gastroenterologist. J Ped Gast Nutr. 2008;47:S49-52

10. Fogg MI, Spergel JM. Management of food allergies. Expert Opin Pharmacother. 2003:4:1025-37.

11. Sicherer SH, Sampson HA. Food Allergy. J Allergy Clin Immunol. 2010;125:S116-25.

12. Liacouras CA, Furuta GT, Hirano I, et al. Eosinophilic esophagitis: Updated consensus recommendations for children and adults. J Allergy Clin Immunol. 2011;128:3-20.

13. Jimenez SG, Catto-Smith AG. Impact of day-case gastroscopy on children and their families. J Gast Hepatol. 2008;23:379-84.

14. Ammar MS, Pfefferkorn MD, Croffie JM, et al. Complications after outpatient upper Gl endoscopy in children: 30-day follow-up. Am J Gastroenterol. 2003;98:1508-11.

15. Volonaki E, Sebire NJ, Borrelli O, et al. Gastrointestinal Endoscopy and Mucosal Biopsy in the First Year of Life: Indications and Outcome. J Pediatr Gastro Nutr. 2012;55:62-5.

16. Sebire NJ, Ramsay A, Smith W, Malone M, Risdon RA. Lamina propria eosinophil density in paediatric gastrointestinal mucosal biopsies. J Pathol. 2002;198:25a.

17. Papadopoulou A, Koletzko S, Heuschkel R, et al. Management guidelines of Eosinophilic Esophagitis in Childhood. J Pediatr Gastrol Nutr. 2014;58:107-88.

18. Atkins D, Furuta GT. Mucosal immunology, eosinophic esophagitis, and other intestinal inflammatory diseases. J Allergy Clin Immunol. 2010;125:S255-61.

19. Melville D, da Silva MS, Young J, et al. Postprocedural effects of gastrointestinal endoscopy performed as a day case procedure in children: implications for patient and family education. Gastroenterology Nursing. 2007:30:426-34

20. National Institute for Health and Care Excellence (NICE). CG116 Food allergy in children and young people: NICE guideline 2012 http://guidance.nice. org.uk/CG116

21. Venter C, Brown T, Shah N, et al. Diagnosis and management of non-lgEmediated cow's milk allergy in infancy - a UK primary care practical guide. Clin Transl Allergy. 2013;3:23.

22. Chandrasekharan B, Nezami BG, Srinivasan S. Emerging neuropeptide targets in inflammation: NPY and VIP. Am J Physiol Gastrointest Liver Physiol. 2013;304:G949-57.

23. Wood JD. Enteric neuroimmunophysiology and Pathophysiology. Gastroenterol. 2004:127:635-57.

24. Tunis MC, Marshall JS. Toll-Like Receptor 2 as a Regulator of Oral Tolerance in the Gastrointestinal Tract. Mediators Inflamm. 2014; 606383.

\section{Submit your next manuscript to BioMed Central and take full advantage of:}

- Convenient online submission

- Thorough peer review

- No space constraints or color figure charges

- Immediate publication on acceptance

- Inclusion in PubMed, CAS, Scopus and Google Scholar

- Research which is freely available for redistribution

Submit your manuscript at www.biomedcentral.com/submit 